\title{
RELACIÓN ENTRE LA PRESIÓN TRIBUTARIA Y LA RECAUDACIÓN DE LOS PRINCIPALES TRIBUTOS EN AREQUIPA (IMPUESTO GENERAL A LAS VENTAS E IMPUESTO A LA RENTA)
}

\section{THE RELATION BETWEEN TAX BURDEN AND THE COLLECTION OF THE MAIN TAXES IN AREQUIPA (GENERAL SALES TAX AND INCOME TAX)}

Gutiérrez Anco, Mary Carmen, Arce Vilca, Solange, Condori Suyo, Karina Chambi Tuero, Leslie Areliz , Huayta Chambi, Lizeth María Universidad Católica San Pablo, Arequipa, Perú

\section{Resumen:}

En el presente artículo se muestra los resultados obtenidos de un estudio estadístico y correlacional entre la Presión Tributaria ejercida en Perú y la recaudación de los principales tributos de la región de Arequipa (Impuesto General a las Ventas e Impuesto a la Renta).

Se tomó como muestra una serie de cifras anuales obtenidas de la base de datos del INEI, MEF y SUNAT en el período de 2004 a 2012, extraídos en una ficha de recolección de datos de doble entrada. Los cuales se compararon estadísticamente y se sometieron al Método de Correlación de Pearson.

Se obtuvo una relación media entre Presión Tributaria en Perú - Impuesto a la Renta en la región Arequipa y Presión Tributaria en Perú - Impuesto General a las Ventas en la región de Arequipa; obteniendo como resultado 0.02996 y 0.01410 respectivamente.

Es decir, si se da un incremento en la variable de Presión Tributaria, también se dará un incremento proporcional en la variable de principales tributos recaudados en Arequipa. 
Palabras clave: Presión tributaria, inflación, PBI

\section{Summary:}

This article shows the results of a statistical and correlative study between tax pressure exercised in Peru and collection of major taxes in the region of Arequipa (General Sales Tax and Income Tax).

A series of annual figures obtained from the database INEI SUNAT MEF and from the period 2004 to 2012 were taken as a sample and extracted on a double entry data collection sheet. These figures were statistically compared and subjected to the Pearson correlation method.

We obtained an average ratio between the Tax Burden in Peru - Income Tax, and the Tax Burden in the region of Arequipa, Peru - General Sales Tax, resulting in 0.02996 and 0.01410 respectively.

It can be concluded that if an increase occurs in the Tax Burden variable, there will be a proportional increase in the variable of main taxes collected in Arequipa.

Key Words: Tax burden, inflation, gross domestic product

\section{Introducción}

Todos somos testigos de los cambios que se están dando actualmente en Perú (con un crecimiento sostenido); no solo en los ámbitos cultural, político, tecnológico o social si no también en lo económico y tributario.

Según el Ministerio de Economía y Finanzas (MEF), Perú continuará en un entorno muy incierto y con riesgos; aun así, podrá mantenerse como una economía dinámica y estable, estimándole un crecimiento anual de 6,o\% - 6,5\% (MEF M. d., 2012).

Según el economista Pedro Pablo Kuczynski, todo ello se debe básicamente a dos pun- tos. El primero es histórico; refiriéndose al mejoramiento que está teniendo gran parte de la población (desde hace ya varios años) en cuanto a su desenvolvimiento en los diferentes ámbitos como el comercial, laboral o social, impulsados por su creatividad y emprendimiento. El segundo es el hecho de que en los últimos 20 años hemos ido mejorando en nuestras políticas económicas, las cuales nos han permitido estabilizar las finanzas públicas, reducir el endeudamiento del gobierno, promover el crédito interno y disminuir la inflación al nivel de los países avanzados. Asimismo, en la última década, Perú ha tenido una gran apertura a mercados globales (exportaciones); alcanzando hasta 
una participación del 25\% del producto bruto interno (PBI), incrementando nuestras oportunidades e ingresos (Kuczynski, 2013).

Todo este crecimiento también se ve reflejado en datos estadísticos, destacando Perú con un crecimiento promedio anual del 5\%, a comparación con otros países de América Latina (Ministerio de Economía y Finanzas, 2012).

A consecuencia de ello, el Estado ha asumido con mayor cautela y esfuerzo la recaudación y administración de nuestros recursos; implementando y reforzando una serie de políticas, normas, mecanismos y herramientas de gestión y control.

En Perú, por lo general, la implementación de políticas económicas y tributarias se basa en una serie de indicadores, destacando entre ellos la Presión Tributaria.

Dicha presión es un indicador cuantitativo, la cual se obtiene relacionando nuestros ingresos tributarios totales con nuestro producto bruto interno (PBI) en períodos de igual magnitud; entendiéndose como PBI el valor total de la producción de bienes y servicios de un país en un determinado período, independientemente de la propiedad de los activos productivos.

Específicamente, determina el peso que tienen los impuestos en una economía; y con ello nos permite medir la intensidad con la que un país grava a sus contribuyentes a través de las tasas impositivas; como el Impuesto a la Renta (IR), Impuesto General a las Ventas (IGV), Impuesto Selectivo al
Consumo (ISC) o el impuesto a las importaciones (aranceles); siendo estos los principales impuestos que componen los ingresos tributarios del Gobierno Central en Perú (Anónimo, 2013).

La Superintendencia Nacional de Aduanas y de Administración Tributaria (SUNAT) es una entidad pública descentralizada del sector de Economía y Finanzas, dotada de personería jurídica de derecho público; siendo uno de los principales protagonistas que ayuda a determinar la Presión Tributaria de nuestro país; encargada principalmente de gestionar integradamente el cumplimiento de las obligaciones tributarias y aduaneras y de facilitar el comercio exterior, de forma eficiente, transparente y legal (SUNAT S. N., 2013).

En base a este indicador, el Estado puede tomar decisiones económicas y financieras; aplicando políticas tributarias pertinentes y accesibles, con el propósito de que la carga fiscal sea distribuida equitativamente entre todos los participantes (contribuyentes). Sin embargo, a pesar de su tendencia al alza, su Presión Tributaria aún sigue estando entre las más bajas de la región.

Según la Organización para la Cooperación y Desarrollo Económico (OCDE), Perú se encuentra en una situación de expansión económica moderada, reflejándose en el incremento que ha logrado en su Presión Tributaria en la última década, pasando de $12.39 \%$ en el año 2000 al $16.02 \%$ en el año 2012; originado mejores condiciones macroeconómicas, cambios en los regímenes tributarios, y fortalecimiento en la gestión 
tributaria (Cayatopa Rivera, Capcha Carhuamaca, \& Orbegozo Flores, 2012).

Diversos analistas y funcionarios públicos han debatido sobre la situación tributaria del país y pronostican que dicho indicador se incrementará por lo menos en un punto y medio porcentual; y para lograrlo se requerirán medidas de ampliación de la base tributaria, con una reducción en la evasión y elusión tributaria (Gonzales de Olarte, 2010).

Coincidiendo de la misma forma, la Sra. Tania Quispe (jefe de la SUNAT), detalló que la estrategia para incrementar la base tributaria se concentrará principalmente en el mercado interno, debido a que gran parte de las exportaciones (sobre todo las mineras), y los precios internacionales del cobre, oro, gas y petróleo vienen reportando una tendencia a la baja desde 2012 (Quispe Mansilla, 2013).

Es por ello que la ampliación de la base tributaria, de manera permanente, está directamente relacionada con un buen desempeño del IGV, el cual proyecta un crecimiento del 6.9\% y del IR, hasta un 4.5\% (MEF M. d., 2013).

La base tributaria se refiere a la masa potencial de ingresos que se obtienen de los distintos sectores de fuentes de riqueza (Gonzales De Olarte, 2010).

Al mes de diciembre del 2012, el padrón de contribuyentes es aproximadamente 6,2 millones, y se explica básicamente por el incremento del segmento de los Medianos y Pequeños Contribuyentes (MEPECOS). A comparación del año anterior, representa un crecimiento del $11 \%$, incrementando la base con más de 600 mil nuevos contribuyentes registrados (SUNAT, 2013).

Los tributos son considerados como fuente principal de los ingresos de un país dirigido a inversiones como salud, educación, vivienda, infraestructura y demás sectores. Es por ello que su importancia radica en la capacidad de recaudación.

El Impuesto General a las Ventas e Impuesto a la Renta son los principales tributos que intervienen en la base tributaria; puesto que están presentes en cada una de las fases de las transferencias que se registran en el circuito económico que recorren todos los productos y servicios (Saenz Rabanal, 2010).

Según el Código Tributario, el Impuesto a la Renta es un tributo que se determina anualmente; se clasifica por categorías y regímenes. Grava las rentas provenientes de la explotación de un capital (primera y segunda categoría); del trabajo realizado de forma independiente o dependiente (cuarta y quinta categoría); y las obtenidas de la aplicación conjunta de ambos factores (tercera categoría o empresarial).

Siendo la renta de tercera categoría la de mayor participación, principalmente en el régimen general; ya que no presenta ningún tipo de restricciones y considera todo tipo de actividades como el comercio, la industria, la manufactura y diversos servicios (Cayatopa Rivera, Capcha Carhuamaca, \& Orbegozo Flores, 2012).

El Impuesto General a las Ventas es un impuesto plurifásico, el cual se encuentra 
estructurado en base a la técnica del valor agregado; se liquida mensualmente y su tasa es del $16 \%$.

Todo este crecimiento sostenido en Perú, depende fundamentalmente de la participación progresiva de sus componentes, es decir, de sus provincias; quienes han aprovechado eficientemente las oportunidades que les ha presentado el mercado, tanto interno como externo.

Las 10 principales ciudades que intervienen en este progreso son Lima, Arequipa, Trujillo, Ilo, Chiclayo, Tacna, Cusco, Ica, Huancayo y Piura (Perú Económico, 2012).

La ciudad de Arequipa tiene gran participación en este progreso; debido a que esta ciudad está viviendo un crecimiento económico formidable, impulsado por la bonanza económica del país, basado exclusivamente en factores comerciales y por la mayor disponibilidad de recursos; destacando principalmente en los rubros de comercio, manufactura, servicios y construcción.

Al mes de mayo de 2013, la recaudación tributaria en Arequipa se incrementó en un $12.82 \%$, a comparación del mismo periodo del año pasado, según información emitida por la SUNAT. Además, el IGV recaudado fue el tributo con mayor crecimiento llegando al $20,40 \%$.

Los empresarios arequipeños han estimado que para los años en adelante, la región tendrá un crecimiento económico superior al del promedio nacional, por consumo local, gastos del gobierno central y regional, inversiones privadas y exportaciones principalmente.

\section{Método}

\section{Muestra}

La muestra estuvo conformada por datos cuantitativos, siendo estos los montos anuales correspondientes a los ingresos tributarios por el Impuesto General a las Ventas e Impuesto a la Renta de la región Arequipa, y a las cifras porcentuales que compete la Presión Tributaria en Perú.

Como criterio, se consideró que los datos provengan de fuentes fehacientes y fidedignas, extrayéndolos de informes presentados por el Instituto Nacional de Estadísticas e Informática (INEI), Ministerio de Economía y Finanzas (MEF) y Superintendencia Nacional de Aduanas y Administración Tributaria (SUNAT).

Se consideraron los datos presentados entre el año 2004 y el 2012.

\section{Instrumento}

Como instrumento de investigación se utilizó una ficha de recolección de datos.

Esta ficha nos permitió reunir toda la información requerida de los períodos solicitados (2004 - 2012); en relación al porcentaje de la Presión Tributaria de Perú y los montos de la recaudación del Impuesto General a las Ventas e Impuesto a la Renta de Arequipa.

Este instrumento fue constituido por un cuadro de doble entrada, refiriéndonos a los periodos tomados en el lado vertical; mien- 
tras que para el lado horizontal, se tomaron en cuenta las variables en estudio: Presión Tributaria de Perú, Impuesto General a las Ventas e Impuesto a la Renta de Arequipa.

\section{Procedimiento}

En primer lugar se buscaron todas las fuentes mencionadas donde se encontró la información en cuestión.

Una vez obtenidos todos los datos requeridos se procedió a recoger la información en las fichas de recolección de datos.

Seguidamente se les sometió a un análisis estadístico de correlación y se procesaron mediante el Método de Correlación de Pearson, con el objetivo de determinar la relación existente entre las variables, emitiéndose finalmente un informe.

\section{Resultados}

Para el procesamiento de los datos se utilizó pruebas paramétricas de correlación (Método de Correlación de Pearson), respecto a las variables cuantitativas.

Se analizó las variables: Presión Tributaria del Perú y recaudación del Impuesto General a las Ventas e Impuesto a la Renta de la región Arequipa.

Se observó que la Presión Tributaria en Perú ha ido incrementándose con el transcurso de los años, logrando extenderse desde un $13.1 \%$ para el año 2004 hasta un $16 \%$ para el año 2012.

\section{Gráfico 1}

Presión tributaria de Perú $2004-2012$

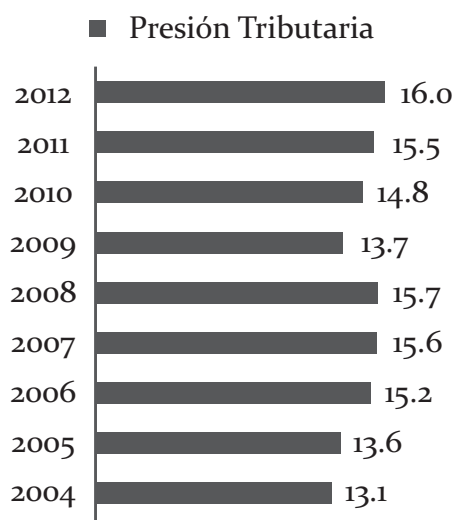

En la Gráfico 1 se muestra la evolución de la Presión Tributaria de Perú en la última década.

Pudimos apreciar que tal variable ha tenido un incremento progresivo año tras año; siendo el año 2009 el único en el que ha disminuido considerablemente.

Observamos las más altas cifras porcentuales en los años 2007, 2008, 2011 y 2012.

De la misma forma, se observó que hubo valores menores en los primeros años del análisis, como en el periodo desde el año 2000 al 2007. 


\section{Gráfico 2}

Impuesto a la Renta de Arequipa

$2004-2012$

En miles de soles

n IR

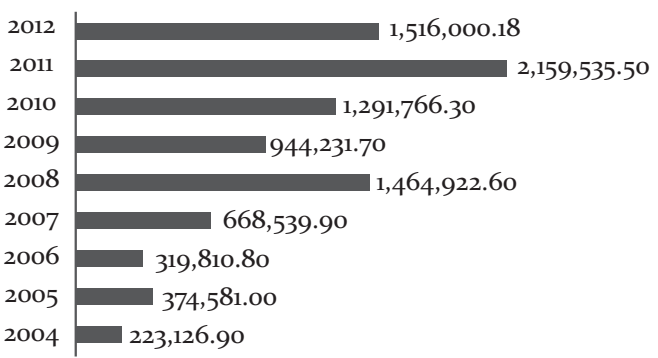

Según el Gráfico 2, se observó que en la región Arequipa se obtuvo mayores ingresos tributarios por parte del Impuesto a la Renta en los años 2008, 2010, 2011 y 2012.

Por el contrario, también se percibió que los valores más bajos se encuentran en los años 2004, 2005, 2006 y 2007.

La recaudación del Impuesto a la Renta en la región Arequipa no mostró una tendencia; es decir, sus valores son variables.

\section{Gráfico 3}

Impuesto General a las Ventas de Arequipa $2004-2012$

En miles de soles

- IGV

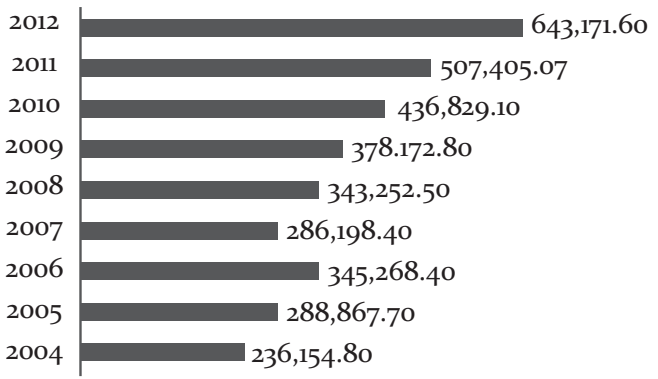

En el Gráfico $\mathrm{N}^{\circ} 3$ se distinguió, de igual forma que la recaudación del Impuesto a la Renta, cifras mayores en los períodos 2009, 2010, 2011 y 2012.

También se contempla que este tipo de impuesto en la región Arequipa posee una tendencia al alza, incrementándose en la última década aproximadamente en 407,016.80 miles de soles.

Tabla 1

Datos de la Presión Tributaria de Perú -Impuesto General a las Ventas e Impuesto a la Renta de Arequipa, 2004 a 2012

\begin{tabular}{|c|c|c|c|}
\hline \multicolumn{2}{|c|}{} & \multicolumn{2}{|c|}{ En miles de nuevos soles } \\
\hline Año & Presión Tributaria & \multicolumn{1}{c|}{ IR } & \multicolumn{1}{c|}{ IGV } \\
\hline 2004 & 13,1 & $223,126.90$ & $236,154.80$ \\
\hline 2005 & 13,6 & $374,581.00$ & $288,867 \cdot 70$ \\
\hline 2006 & 15,2 & $319,810.80$ & $345,268.40$ \\
\hline 2007 & 15,6 & $668,539 \cdot 90$ & $286,198.40$ \\
\hline 2008 & 15,7 & $1,464,922.60$ & $343,252.50$ \\
\hline 2009 & 13,7 & $944,231.70$ & $378,172.80$ \\
\hline 2010 & 14,8 & $1,291,766.30$ & $436,829.10$ \\
\hline 2011 & 15,5 & $2,159,535.50$ & $507,405.07$ \\
\hline 2012 & 16,0 & $1,516,000.18$ & $643,171.60$ \\
\hline
\end{tabular}

Comparando los datos extraídos en un cuadro de doble entrada, se observó que la Presión Tributaria ejercida en Perú ha ido incrementando conjuntamente con ambos impuestos (IGV e IR) recaudados en la región Arequipa.

Con la aplicación del Método de Correlación de Pearson, se obtuvo una relación positiva en ambos casos; Presión Tributaria - Impuesto a la Renta y Presión Tributaria - Im- 
puesto General a las Ventas; dando como resultado 0.02996 y 0.01410 respectivamente.

Dicho resultado obtenido indica la existencia de una relación perfectamente positiva en ambos casos; es decir que cuando al variar la primera, la segunda varía en las mismas proporciones y en la misma dirección.

\section{Discusión}

Hoy en día en Perú se han observado diversos cambios, los cuales han afectado las decisiones en cuanto a la fijación de políticas económicas y financieras, basándose en indicadores.

La Presión Tributaria es uno de los principales indicadores que se toma en cuenta para determinar políticas que rigen el comportamiento en Perú.

Este indicador determina el peso que tienen los impuestos en la economía de Perú; y es en base a ello que se fija la intensidad con la que se debería gravar a todos los contribuyentes a través de los tributos; siendo los de mayor recaudación el Impuesto a la Renta (IR) e Impuesto General a las Ventas (IGV).

Según los resultados estadísticos obtenidos, se pudo destacar que sí hay relación directa entre la Presión Tributaria ejercida en Perú con los principales ingresos tributarios recaudados en la región Arequipa (IGV e IR).

A partir del año 2009, se ha observado un crecimiento continuo en todas las variables. Este empuje se inicia después de la crisis económica mundial de 2008.
La situación económica y financiera de nuestro país se ha estabilizado y se está preparando ante cualquier otra circunstancia crítica.

Es por ello que fijar efectivamente las políticas y normas en la gestión puede ocasionar incremento de oportunidades, mayor protección o mejoramiento de la calidad de vida de toda la población.

Comparando los resultados obtenidos con los factores externos e internos a los que se enfrentaba nuestro país y región, se infirió que se tuvo un incremento en las variables debido al crecimiento económico y buenas decisiones, materializándose en un incremento de obras y servicios para bien de toda la población.

Arequipa es una de las regiones que ha alcanzado un crecimiento considerable en cuanto a la recaudación de tributos en la última década, mejorando la calidad de vida de sus pobladores.

De acuerdo a los resultados obtenidos por la aplicación del Método de Pearson, se concluye que la región Arequipa va de la mano con las políticas tomadas por el Gobierno Central.

Con todo lo expuesto, se dedujo que las políticas internas que toman las entidades en la región Arequipa son relativamente efectivas, debido a que el resultado (al aplicar el Método de Correlación de Pearson) no se encuentra muy cercano a la unidad. 


\section{Referencia}

Perú Económico. (2012). Recuperado en octubre de 2012, de Top 10: Ciudades con mejor calidad de vida: http://perueconomico.com/ediciones/74/articulos/1337

Anónimo. (2013). Instituto Peruano de Economía. Recuperado en agosto de 2013, de Presión Tributaria: http://ipe.org.pe/ content/presion-tributaria

Cabanellas de Torres, G. (1979). Diccionario Enciclopédico de Derecho Usual. Argentina, Buenos Aires: Heliasta.

Cárdenas, M., \& Rozo, S. (2009). Informalidad social, problemas y soluciones - Desarrollo y Sociedad.

Cayatopa Rivera, L. E., Capcha Carhuamaca, C., \& Orbegozo Flores, R. M. (2012). "Evolución de la relación recaudatoria entre el Impuesto a la Renta, Impuesto a las Transacciones Financieras, Impuesto General a las Ventas e Impuesto Selectivo al Consumo (2004 - 2011)". Universidad Católica Sedes Sapientae.

García, E. (2005). “Competitividad e innovación en la Micro y Pequeña Empresa?" . Valencia .

Gonzales de Olarte, E. (18 de marzo de 2010). Economía Peruana. Recuperado en diciembre de 2012, de ¿Por qué la presión tributaria no sube?: http://blog. pucp.edu.pe/item/91967/porque-la-presion-tributaria-no-sube
Gonzales De Olarte, E. (18 de marzo de 2010). Economía Peruana. Recuperado el 30 de octubre de 2012, de ¿Por qué la presión tributaria no sube?: http://blog. pucp.edu.pe/item/91967/porque-la-presion-tributaria-no-sube

Kuczynski, P. P. (31 de Marzo de 2013). ¿Porqué crece el Perú? Correo.

MEF, M. d. (2012). Marco Macroeconómico Multianual entre el 2012 - 2015. Lima.

MEF, M. d. (2013). Marco Macroeconómico Multianual 2013 - 2015. Lima.

Ministerio de Economía y Finanzas. (2012). Informe Anual 2011 - Buscando un crecimiento equitativo y equilibrado. Lima.

Montoya , M. (1998). "Derecho Comercial". Lima: Editora jurídica Grijley E.I.R.L.

Quispe Mansilla, T. (1o de mayo de 2013). Recaudación tributaria del impuesto a la renta cayó 8,2\% durante el 2012. (E. Comercio, Entrevistador)

Sáenz Rabanal, M. J. (2010). “Tratamiento del Crédito Fiscal”. VII Jornadas Nacionales de Derecho, Perú.

SUNAT. (og de enero de 2013). Nota de Prensa $N^{\circ}$ oo6-2013.Lima.

SUNAT, S. N. (2013). SUNAT.Recuperado el 10 de septiembre de 2013, de http://unete. sunat.gob.pe/index.php?option=com_co ntentEview $=$ articleEid $=177 \mathcal{E}$ Itemid $=74$

WordReference. (s.f.). Diccionario. Recuperado en agosto de 2013, de http://www. wordreference.com/definicion/ 